\title{
Electron-Vibration Interaction in the Presence of a Switchable Kondo Resonance Realized in a Molecular Junction
}

\author{
D. Rakhmilevitch, ${ }^{1}$ R. Korytár, ${ }^{2}$ A. Bagrets, ${ }^{2,3}$ F. Evers, ${ }^{2,4}$ and O. Tal ${ }^{1}$ \\ ${ }^{1}$ Department of Chemical Physics, Weizmann Institute of Science, Rehovot 761001, Israel \\ ${ }^{2}$ Institute of Nanotechnology, Karlsruhe Institute of Technology, Karlsruhe 76128, Germany \\ ${ }^{3}$ Steinbuch Centre for Computing, Karlsruhe Institute of Technology, Karlsruhe 76128, Germany \\ ${ }^{4}$ Institut Für Theorie der Kondensierten Materie, Karlsruhe Institute of Technology, Karlsruhe 76128, Germany
}

(Received 22 June 2014; published 5 December 2014)

\begin{abstract}
The interaction of individual electrons with vibrations has been extensively studied. However, the nature of electron-vibration interaction in the presence of many-body electron correlations such as a Kondo state has not been fully investigated. Here, we present transport measurements on a Copper-phthalocyanine molecule, suspended between two silver electrodes in a break-junction setup. Our measurements reveal both zero bias and satellite conductance peaks, which are identified as Kondo resonances with a similar Kondo temperature. The relation of the satellite peaks to electron-vibration interaction is corroborated using several independent spectroscopic indications, as well as ab initio calculations. Further analysis reveals that the contribution of vibration-induced inelastic current is significant in the presence of a Kondo resonance.
\end{abstract}

DOI: 10.1103/PhysRevLett.113.236603

The Kondo effect is one of the most intensely studied many body phenomena, both experimentally and theoretically [1,2]. Although the basic Kondo exchange process was initially described in the 1960s and 1970s [3-6], new fascinating aspects are emerging to this day, including the orbital Kondo effect [7,8], the influence of Ruderman-Kittel-Kasuya-Yosida (RKKY) interactions $[9,10]$, and the interplay between Kondo physics and superconductivity [11]. The Kondo effect has also been observed in molecular junctions, in which a molecule is located between two metallic electrodes [12-15]. In this system the localized magnetic moment on the molecule is screened by an exchange process with the itinerant electrons of the electrodes. This process leads to an enhanced density of states at the Fermi energy due to the emergence of the Abrikosov-Suhl resonance, observed in differential conductance measurements as a peak at zero-bias voltage. With the aid of molecular junctions, intriguing aspects of Kondo physics were explored such as the underscreened Kondo effect [16,17], the distribution of Kondo screening over different submolecular segments [18], and the influence of nearby molecules [19].

One of the attractive features of single-molecule junctions is the ability to couple molecular vibrations to conduction electrons [20]. In this context, molecular junctions are a natural platform for studying electromechanical interactions in the quantum regime. When an applied voltage across a molecular junction exceeds the energy of a given mode of vibration, this mode can be excited by the injected electrons. For a weak electronvibration interaction in the off-resonance regime, an electron can scatter forward by exciting a vibration mode,
PACS numbers: 72.15.Qm, 73.23.-b, 81.07.Nb, 85.65.+h

resulting in an additional contribution to the current. Experimentally, this inelastic contribution manifests as a stepwise increase in the measured differential conductance at a voltage equivalent to the vibration energy [21]. This spectroscopic feature is commonly used to indicate the presence of a molecule in the junction as well as to chemically identify the molecule [22,23].

While a large body of experimental and theoretical findings addressing the interaction between independent electrons and vibrations is available, the understanding of electron-vibration interaction in correlated-electron systems has remained limited. Previously reported experiments detected a zero-bias Kondo peak in the differential conductance accompanied by satellite peaks at finite bias [24-26]. These features were often ascribed to the vibration assisted Kondo resonance for which the excess energy of the electrons due to a finite voltage is compensated by activating a vibration mode. However, establishing an explicit relation between vibrations and the Kondo effect remains an elusive challenge and the properties of electronvibration interaction in the presence of such a many-body electron state were not addressed experimentally despite many theoretical studies [27-29].

In this Letter, we use molecular junctions based on silver $(\mathrm{Ag})$ electrodes and copper-phthalocyanine $(\mathrm{CuPc})$ molecules to identify the spectroscopic signature of electronvibration interaction in the presence of a Kondo effect. This is confirmed by the evolution of satellite peaks to vibration-induced conductance steps when the Kondo resonance is suppressed. Furthermore, the nonmonotonic response of the satellite peaks to junction elongation reveals a typical signature of a vibration mode, as verified 
by calculations. We find (i) a dominant influence of the Kondo effect on the evolution of the satellite peaks as a function of temperature and (ii) a substantial conductance contribution due to the vibration assisted Kondo effect with respect to the conductance of the zero-bias Kondo resonance.

The studied molecular junctions were fabricated in a mechanically controllable break-junction (MCBJ) setup [30] allowing a stable, subatomic control over the distance between the electrodes. Such changes were used before to modify both the manifestation of Kondo physics [25] and the activation of vibrations in atomic scale junctions $[23,31,32]$. The break junction samples were made of a silver wire segment (99.997\%, GoodFellow), partially cut in the middle and attached to a flexible substrate. The samples were mounted into a vacuum chamber that was pumped and cooled to cryogenic temperature. The weak spot in the center of the wire was broken in cryogenic vacuum to expose ultraclean electrode apices. The bare silver atomic junction was characterized by recording conductance vs junction elongation for thousands of repeatedly formed contacts (see Supplemental Material [33]) and inelastic spectroscopy measurements [Fig. 1(a), blue]. CuPc molecules (99.95\%, Sigma-Aldrich, further purified in situ) were introduced to the contact by controlled sublimation from a local source. The formation of molecular junctions was monitored by detecting conductance values below $\sim 1 G_{0}$, the typical conductance of a bare silver atomic contact, and verified using inelastic
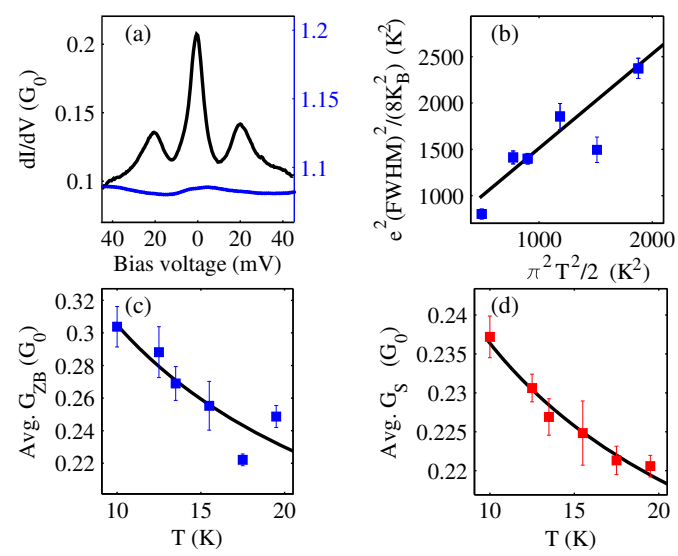

FIG. 1 (color online). (a) Typical differential conductance spectrum obtained before (blue) and after (black) admission of $\mathrm{CuPc}$ molecules to the silver atomic junction. (b) Full width at half maximum (FWHM) of the zero-bias peak as a function of temperature. The curve is a fit to Eq. (2). (c) Average conductance of the zero-bias peak $\left(G_{Z B}\right)$ as a function of temperature. The curve is a fit to Eq. (1). (d) Average conductance of the satellite peaks $\left(G_{S}\right)$ as a function of temperature. The curve is a fit to Eq. (1) combined with an offset due to the background conductance of the zero-bias peak. Error bars indicate the standard error of the data set obtained on an ensemble of junctions at each temperature. spectroscopy to detect molecular vibrations in bias voltages above the phonon spectrum of the metal [47].

After admission of molecules into the junction, a prominent zero-bias peak was observed in $30 \%$ of the independently formed junctions, in most cases accompanied by two satellite peaks at finite bias [Fig. 1(a), black]. Since CuPc is known to have a spin $1 / 2$ both in the gas phase as well as in epitaxial layers [48], and Kondo resonance was observed for $\mathrm{CuPc}$ adsorbed on a silver substrate in STM experiments [49], the Kondo effect is a possible source for the observed peak at zero bias. In order to test this possibility, we collected differential conductance spectra of 186 independently formed junctions measured at different temperatures and magnetic fields. The average conductance at zero bias and the full width at halfmaximum (FWHM) of the zero-bias peak follow the appropriate functional dependence with temperature for a spin 1/2 Kondo impurity [50,51]:

$$
\begin{gathered}
G(T, V=0)=\frac{\alpha}{\left[1+\left(\frac{T}{T_{K}}\right)^{2}\left(2 \frac{1}{0.22}-1\right)\right]^{0.22}} \\
\frac{e^{2}}{8 k_{B}^{2}}(\mathrm{FWHM})^{2}=(\pi T)^{2} / 2+T_{K}^{2}
\end{gathered}
$$

with Kondo temperatures of $T_{K}=21 \pm 5 \mathrm{~K}$ and $T_{K}=$ $22 \pm 5 \mathrm{~K}$, respectively [see the fitting in Figs. 1(b) and $1(\mathrm{c})]$.

As shown in Fig. 2(a), application of an external magnetic field perpendicular to the junction axis results in a notable increase in the average FWHM of the zerobias peak. While a clear peak splitting was not observed due to the high temperature, Fig. 2(b) shows that at a magnetic field of $8.5 \mathrm{~T}$, the zero-bias peak can be fitted by the sum of two Lorentzian functions, each with a FWHM of $6 \mathrm{mV}$, which are separated by $3.14 \mathrm{mV}$ as expected for single molecule junctions with spin $1 / 2$ Kondo resonance [12]. The evolution of the zero-bias peak as a function of temperature and applied magnetic field indicates that spin $1 / 2$ Kondo correlations are the source of the zerobias peak.

The appearance of satellite peaks accompanying the zero-bias Kondo resonance suggests that the Kondo effect plays a dominant role in the formation of these features. To examine our hypothesis, we analyzed the influence of temperature on the properties of the satellite peaks. As can be seen in Fig. 1(d), the decrease in the conductance of the satellite peaks with increasing temperature is described remarkably well by Eq. (1). Based on this fitting, the extracted Kondo temperature for the satellite peaks is $25 \pm 5 \mathrm{~K}$, which is similar to the Kondo temperature obtained for the zero-bias peak. We note that this analysis excludes the mechanism of a vibration-induced two-level system as the origin of the finite bias peaks $[52,53]$. 

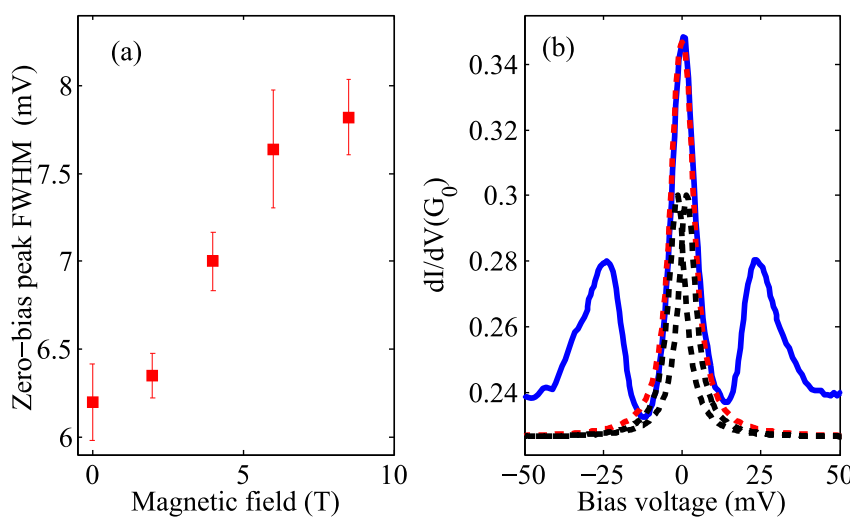

FIG. 2 (color online). (a) Dependence of the average zero-bias peak FWHM on external magnetic field. (b) Fitting of a zero-bias peak, obtained in an external magnetic field of $8.5 \mathrm{~T}$, by the sum of two Lorentzians. Both the separation and the FWHM result from automatic fitting without any preset boundaries. The sum (dashed, red line) of the individual Lorentzians (dashed, black line) with a FWHM of $6 \mathrm{mV}$, separated by $3.14 \mathrm{mV}$, fits with the zero-bias peak with a FWHM of $8.5 \mathrm{mV}$.

In order to further study the origin of the satellite peaks, we focused on differential conductance spectra measured on an ensemble of independently formed molecular junctions. In 31 junctions, when the zero-bias peak was suppressed, as seen in Fig. 3(a), we observed conductance steps at voltage values corresponding to the satellite peaks [Fig. 3(b)]. Such an increase in the conductance as a function of voltage is a typical signature of vibration
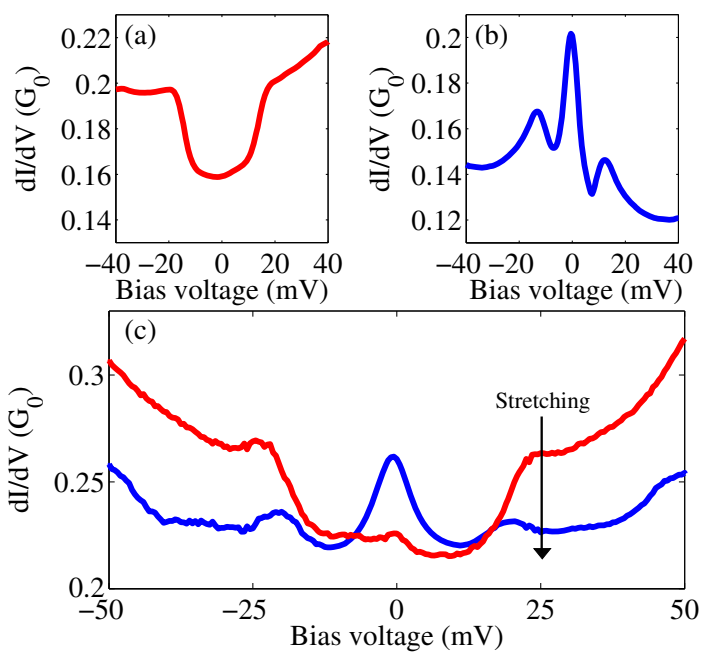

FIG. 3 (color online). Differential conductance curves vs applied voltage, measured on independently formed $\mathrm{Ag} / \mathrm{CuPc}$ molecular junctions. (a) Finite-bias steps appear when the zero-bias peak is absent. (b) Finite-bias peaks appear only as satellites in the presence of a zero-bias peak. Note that the differential conductance curves exhibit some asymmetry, probably due to conductance fluctuations [56]. (c) Evolution of a differential conductance spectrum when the junction is stretched by $0.08 \AA$. activation in the off-resonance conduction regime [21]. The presented vibration-induced conductance steps were observed for junctions with different conductance values, indicating that the excitation of the relevant vibration mode is not significantly susceptible to variations in the configuration of the junction. Moreover, evolution of conductance steps into satellite peaks at the same voltages was observed when the junctions were stretched [e.g., Fig. 3(c)], indicating a probable common origin for both features. This evolution can stem from conformational changes in the molecular junction as a result of stretching. Note that similar switching of a zero-bias Kondo effect due to conformational changes was previously observed both in STM [54] and MCBJ experiments [25,55].

To further establish the relation between the satellite peaks and molecular vibrations, we focus on the evolution of differential conductance spectra as a function of junction elongation. Figure 4(a) presents a subset of differential conductance curves for increasing interelectrode distances. As can be seen in Fig. 4(b), when the junction is stretched in steps of $0.25 \AA$ the satellite peaks shift to higher voltage, up to a certain elongation. However, this tendency is inverted when the junction is stretched further; the satellite peaks are then shifted to lower voltage. Remarkably, a similar response to junction stretching can be expected for a vibration mode. Stretching the junction leads to enhancement of the vibration frequency in analogy to the increase of the pitch of a guitar string under tension [23]. This results in an increase of the onset voltage for vibration activation [57]. Further stretching eventually weakens the bonds between the molecule and the electrodes, leading to a
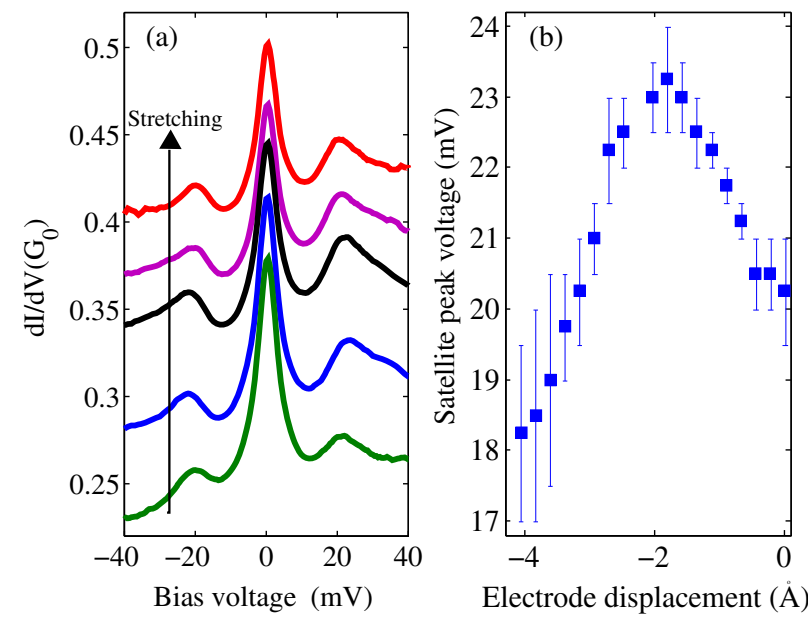

FIG. 4 (color online). (a) A sequence of differential-conductance curves taken at increasing interelectrode distances. The curves are shifted by $0.05 G_{0}$. (b) Averaged voltage of the satellite peaks in each curve [exemplified in (a)] as a function of interelectrode separation. The data of the full stretching sequence is presented in (b), while (a) presents only some of the curves for better clarity. The error bars indicate deviations from the average. 
decrease in the vibration energy and hence a decrease in onset voltage for vibration activation [23,31].

The structure of the junction and the corresponding vibration modes were analyzed using $a b$ initio calculations. In the gas phase the CuPc molecule carries a spin $1 / 2$ moment in a singly occupied orbital $b_{1 g}$, localized near the copper center [58,59]. In contrast to the case of $\mathrm{CuPc}$ adsorbed on a $\mathrm{Ag}$ (100) surface [18], we find no significant charge transfer to the molecule, and the gas-phase electronic structure remains intact even after coupling to the electrode apices [33]. Hence, the experimental observation of the Kondo effect is expected. Out of the 20 candidate vibration modes in the relevant energy window of 10-35 meV, a specific mode was singled out based on (i) the study of vibration coupling to the $b_{1 g}$ orbital in the gas-phase $\mathrm{CuPc}$, and (ii) the analysis of stretching dependence of vibrations in the molecular junction. In essence, the $D_{4 h}$ point group symmetry of the molecule implies a number of selection rules for the electron-phonon matrix elements of the $b_{1 g}$ orbital. Consequently, out of all vibration modes in the relevant energy window, there is only one mode, $A_{1 g}$, [see Fig. 5(a)], with energy of $\hbar \Omega_{0}=31 \mathrm{meV}$ and a sizable electron-phonon coupling $\lambda_{0}=6 \mathrm{meV}$.
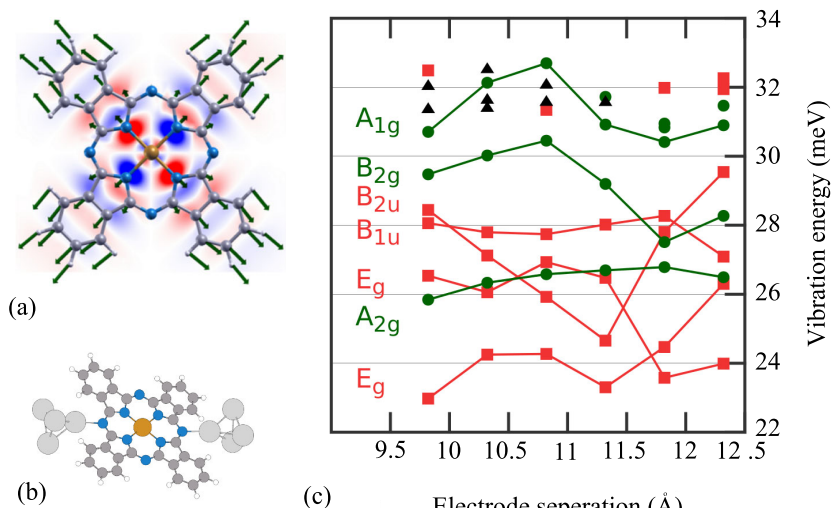

(c) Electrode seperation $(\AA)$

FIG. 5 (color online). Theoretical analysis of molecular vibrations. (a) Ball-and-stick model of $\mathrm{CuPc}$ (hydrogen is white, carbon is gray, nitrogen is blue, and copper is orange). In the background we show the $b_{1 g}$ molecular orbital, that carries the Kondo-screened magnetic moment. Because of selection rules, only the $A_{1 g}$ vibrational mode has a significant electron-phonon coupling matrix element; the atomic displacements (scaled by $\times 10$ ) of this eigenmode are shown with green arrows. (b) Ball-and-stick representation of the structure used to investigate the vibrations of $\mathrm{CuPc}$ in the junction (silver-large gray circles). (c) Flow of vibration frequencies of the junction presented in (b) at 6 successive elongations. The horizontal axis denotes the apex-to-apex distance. For vibrations with distinct character, the Mulliken symbol of the corresponding gas-phase $\mathrm{CuPc}$ mode is shown to the left. Modes with a markedly in-plane character are shown as green circles; modes with a markedly out-of-plane character are shown as red squares; modes without defined character are shown as black triangles.
To inspect the stretching dependence of vibration frequencies, we have considered the relaxed structure in Fig. 5(b), comprising the molecule bound to a pair of $\mathrm{Ag}$ pyramids [33]. In energetically favorable configurations the molecule binds to the Ag electrodes via the nitrogen atoms, restricting the possible orientations of the molecule in the junction. We have increased the apex-to-apex distance in successive steps and traced the behavior of vibrations, as presented in Fig. 5(c). Although the $D_{4 h}$ symmetry is lifted, most of the junction modes retain their gas-phase character. Thus, stretching can be understood as an adiabatic flow of the vibration spectrum. The relevant mode, previously attributed to the gas phase $A_{1 g}$, shows a nonmonotonic energy shift, of at least $2 \mathrm{meV}$, with a pronounced maximum, qualitatively reproducing the experimental observation. The vibration energy is approximately $10 \mathrm{meV}$ higher as compared to the experimental data. We attribute this difference to (a) simplification in our electrode model, which lacks elasticity, and (b) to the more fundamental uncertainty of any density functional theory based estimation of atomic forces due to the approximate nature of the exchangecorrelation functionals. For more information regarding the response of vibration modes with different symmetries to junction stretching, see [33].

To summarize up to this point, our analysis indicates that molecular vibrations coexisting with Kondo screening play a central role in the formation of the satellite peaks. The good agreement of Eq. (1) with our results suggests that within the experimental resolution, the participation of the vibration degree of freedom does not have a significant effect on the temperature dependence of the conductance at the satellite peaks. This weak dependence on temperature holds for $k_{B} T<0.1 \hbar \omega(\hbar \omega \approx 20 \mathrm{meV}$ being the vibration mode energy), as was also shown for the amplitude of vibration-induced steps in the absence of Kondo screening $[60,61]$.

Interestingly, we find that the inelastic conductance due to vibration activation is considerably large in the presence of Kondo screening. In the absence of the Kondo resonance, the fraction of inelastic conductance is approximately determined by the ratio between the height of the conductance step and the zero-bias conductance values $[60,62]$. Performing this analysis on curves exhibiting a step yields an average value of $13 \pm 1 \%$, which is similar to previous observations in single molecule junctions [63]. For junctions exhibiting both zero-bias and satellite peaks, the ratio between the amplitudes of the two types of peaks provides the ratio of the inelastic vibration-assisted Kondo conductance with respect to the elastic Kondo conductance. By analyzing the ensemble of differential conductance curves exhibiting both peaks, we find this ratio to be $26 \pm 3 \%$. This suggest that the electron-vibration interaction can be significant when Kondo screening emerges.

To conclude, we find clear indications for a vibration assisted Kondo effect in $\mathrm{Ag} / \mathrm{CuPc}$ molecular junctions. The 
presented study reveals the properties of electron-vibration interaction in a Kondo correlated electron system. In particular, we find a similar Kondo temperature for the zero-bias Kondo resonance and the vibration assisted Kondo process and a significant inelastic contribution to the Kondo conductance. We hope that our observations will stimulate further investigations, theoretical as well as experimental, of Kondo impurities that are coupled to bosonic baths.

We thank K. L. Narasimhan for his valuable help in developing a molecular evaporation source and M. Bürkle for discussions and sharing with us a development version of TURBOMOLE package, containing an EVIB module. O. T. thanks the Harold Perlman Family for their support and acknowledges funding by the Israel Science Foundation, the German-Israeli Foundation, the Minerva Foundation, the Gerhard Schmidt Minerva Center and the Yeda-Sela Center. A. B. acknowledges support of DFG through the research Grant No. BA 4265/2-1. Computational resources of the HC3 computer cluster at Steinbuch Centre for Computing (KIT) is acknowledged.

[1] A. C. Hewson, The Kondo Problem to Heavy Fermions (Cambridge University Press, Cambridge, England, 1997), ISBN 9780521599474.

[2] L. Kouwenhoven and L. Glazman, Phys. World 14, 33 (2001).

[3] P. W. Anderson, Phys. Rev. 124, 41 (1961).

[4] J. Kondo, Prog. Theor. Phys. 32, 37 (1964).

[5] K. G. Wilson, Rev. Mod. Phys. 47, 773 (1975).

[6] P. Nozières, J. Low Temp. Phys. 17, 31 (1974).

[7] P. Jarillo-Herrero, J. Kong, H. S. J. Van Der Zant, C. Dekker, L. P. Kouwenhoven, and S. De Franceschi, Nature (London) 434, 484 (2005).

[8] M.-S. Choi, R. López, and R. Aguado, Phys. Rev. Lett. 95, 067204 (2005).

[9] Y.-S. Fu, Q.-K. Xue, and R. Wiesendanger, Phys. Rev. Lett. 108, 087203 (2012).

[10] H. B. Heersche, Z. de Groot, J. A. Folk, L. P. Kouwenhoven, H. S. J. van der Zant, A. A. Houck, J. Labaziewicz, and I. L. Chuang, Phys. Rev. Lett. 96, 017205 (2006).

[11] M. R. Buitelaar, T. Nussbaumer, and C. Schönenberger, Phys. Rev. Lett. 89, 256801 (2002).

[12] J. Park, A. N. Pasupathy, J. I. Goldsmith, C. Chang, Y. Yaish, J. R. Petta, M. Rinkoski, J. P. Setna, H. D. Abruña, P. L. McEuen, and D. C. Ralph, Nature (London) 417, 722 (2002).

[13] W. Liang, M. P. Shores, M. Bockrath, J. R. Long, and H. Park, Nature (London) 417, 725 (2002).

[14] L. H. Yu and D. Natelson, Nano Lett. 4, 79 (2004).

[15] S. Wagner, F. Kisslinger, S. Ballmann, F. Schramm, R. Chandrasekar, T. Bodenstein, O. Fuhr, D. Secker, K. Fink, M. Ruben, and H. B. Weber, Nat. Nanotechnol. 8, 575 (2013).

[16] J. J. Parks, A. R. Champagne, T. A. Costi, W. W. Shum, A. N. Pasupathy, E. Neuscamman, S. Flores-Torres,
P. S. Cornaglia, A. A. Aligia, C. A. Balseiro et al., Science 328, 1370 (2010).

[17] N. Roch, S. Florens, T. A. Costi, W. Wernsdorfer, and F. Balestro, Phys. Rev. Lett. 103, 197202 (2009).

[18] A. Mugarza, C. Krull, R. Robles, S. Stepanow, G. Ceballos, and P. Gambardella, Nat. Commun. 2, 490 (2011).

[19] V. Iancu, A. Deshpande, and S.-W. Hla, Phys. Rev. Lett. 97, 266603 (2006).

[20] J. C. Cuevas and E. Scheer, Molecular Electronics: An Introduction to Theory and Experiment, World Scientific Series in Nanotechnology and Nanoscience (World Scientific, Singapore, 2010), ISBN 9789814282581.

[21] B. C. Stipe, M. A. Rezaei, and W. Ho, Science 280, 1732 (1998).

[22] R. C. Jaklevic and J. Lambe, Phys. Rev. Lett. 17, 1139 (1966).

[23] D. Djukic, K. S. Thygesen, C. Untiedt, R. H. M. Smit, K. W. Jacobsen, and J. M. van Ruitenbeek, Phys. Rev. B 71, 161402 (2005).

[24] L. H. Yu, Z. K. Keane, J. W. Ciszek, L. Cheng, M. P. Stewart, J. M. Tour, and D. Natelson, Phys. Rev. Lett. 93, 266802 (2004).

[25] J. J. Parks, A. R. Champagne, G. R. Hutchison, S. FloresTorres, H. D. Abruña, and D. C. Ralph, Phys. Rev. Lett. 99, 026601 (2007).

[26] I. Fernández-Torrente, K. J. Franke, and J. I. Pascual, Phys. Rev. Lett. 101, 217203 (2008).

[27] R.-Q. Wang, Y.-Q. Zhou, and D. Y. Xing, J. Phys. Condens. Matter 20, 045219 (2008).

[28] J. Paaske and K. Flensberg, Phys. Rev. Lett. 94, 176801 (2005)

[29] J. König, H. Schoeller, and G. Schön, Phys. Rev. Lett. 76, 1715 (1996).

[30] C. J. Muller, J. M. van Ruitenbeek, and L. J. de Jongh, Phys. Rev. Lett. 69, 140 (1992).

[31] N. Agraï, C. Untiedt, G. Rubio-Bollinger, and S. Vieira, Phys. Rev. Lett. 88, 216803 (2002).

[32] Y. Kim, H. Song, F. Strigl, H.-F. Pernau, T. Lee, and E. Scheer, Phys. Rev. Lett. 106, 196804 (2011).

[33] See Supplemental Material at http://link.aps.org/ supplemental/10.1103/PhysRevLett.113.236603, which includes Refs. [34-46].

[34] F. Pauly, J. K. Viljas, M. Bürkle, M. Dreher, P. Nielaba, and J. C. Cuevas, Phys. Rev. B 84, 195420 (2011).

[35] R. Vardimon, M. Klionsky, and O. Tal, Phys. Rev. B 88, 161404 (2013).

[36] V. Blum, R. Gehrke, F. Hanke, P. Havu, V. Havu, X. Ren, K. Reuter, and M. Scheffler, Comput. Phys. Commun. 180, 2175 (2009).

[37] E. van Lenthe, E. J. Baerends, and J. G. Snijders, J. Chem. Phys. 101, 9783 (1994).

[38] J. P. Perdew, K. Burke, and M. Ernzerhof, Phys. Rev. Lett. 78, 1396 (1997).

[39] R. Korytár and N. Lorente, J. Phys. Condens. Matter 23, 355009 (2011).

[40] R. Ahlrichs et al., TURBOMOLE v6.6 (development version), program package for $a b$ initio electronic structure calculations, Turbomole GmbH, www.turbomole.com (2014).

[41] A. Schäfer, H. Horn, and R. Ahlrichs, J. Chem. Phys. 97, 2571 (1992). 
[42] K. Eichkorn, O. Treutler, H. Öhm, M. Häser, and R. Ahlrichs, Chem. Phys. Lett. 240, 283 (1995).

[43] P. Deglmann and F. Furche, J. Chem. Phys. 117, 9535 (2002).

[44] P. Deglmann, F. Furche, and R. Ahlrichs, Chem. Phys. Lett. 362, 511 (2002).

[45] P. Deglmann, K. May, F. Furche, and R. Ahlrichs, Chem. Phys. Lett. 384, 103 (2004).

[46] M. Bürkle, J. K. Viljas, T. J. Hellmuth, E. Scheer, F. Weigend, G. Schön, and F. Pauly, Phys. Status Solidi B 250, 2468 (2013).

[47] A. V. Khotkevich and I. K. Yanson, Atlas of Point Contact Spectra of Electron-Phonon Interactions in Metals (Kluwer Academic, Dordrecht, 1995), ISBN 9780792395263.

[48] J. E. Jiang, Functional Phthalocyanine Molecular Materials (Springer, Berlin, Heidelberg, 2010), ISBN 978-3-64204751-0.

[49] A. Mugarza, R. Robles, C. Krull, R. Korytár, N. Lorente, and P. Gambardella, Phys. Rev. B 85, 155437 (2012).

[50] D. Goldhaber-Gordon, J. Göres, M. A. Kastner, H. Shtrikman, D. Mahalu, and U. Meirav, Phys. Rev. Lett. 81, 5225 (1998).

[51] K. Nagaoka, T. Jamneala, M. Grobis, and M. F. Crommie, Phys. Rev. Lett. 88, 077205 (2002).

[52] W. H. A. Thijssen, D. Djukic, A. F. Otte, R. H. Bremmer, and J. M. van Ruitenbeek, Phys. Rev. Lett. 97, 226806 (2006).
[53] A. Halbritter, P. Makk, S. Csonka, and G. Mihály, Phys. Rev. B 77, 075402 (2008).

[54] A. Zhao, Q. Li, L. Chen, H. Xiang, W. Wang, S. Pan, B. Wang, X. Xiao, J. G. Hou, and Z. Qingshi, Science 309, 1542 (2005).

[55] E. Scheer, T. Böhler, A. Edtbauer, S. Egle, M. Erbe, and T. Pietsch, J. Low Temp. Phys. 39, 259 (2013).

[56] B. Ludoph and J. M. van Ruitenbeek, Phys. Rev. B 61, 2273 (2000).

[57] T. Böhler, A. Edtbauer, and E. Scheer, New J. Phys. 11, 013036 (2009).

[58] M.-S. Liao and S. Scheiner, J. Chem. Phys. 114, 9780 (2001).

[59] N. Marom, O. Hod, G. E. Scuseria, and L. Kronik, J. Chem. Phys. 128, 164107 (2008).

[60] M. Paulsson, T. Frederiksen, and M. Brandbyge, Phys. Rev. B 72, 201101 (2005).

[61] L. J. Lauhon and W. Ho, Rev. Sci. Instrum. 72, 216 (2001).

[62] This analysis holds for a single conduction channel as in the examined molecular junction, where the single $s$ valence orbital of the silver atomic contact limits the number of conduction channels to 1 . The presence of a single conduction channel was also verified by calculations.

[63] R. Ben-Zvi, R. Vardimon, T. Yelin, and O. Tal, ACS Nano 7, 11147 (2013). 